\title{
Phosphorylation, Dephosphorylation, and Multiprotein Assemblies Regulate Dynamic Behavior of Neuronal Cytoskeleton: A Mini-Review
}

\begin{abstract}
Natalya Kurochkina ${ }^{1 *}$, Manju Bhaskar², Sharda Prasad Yadav² and Harish C. Pant ${ }^{2 *}$
${ }^{1}$ Department of Biophysics, The School of Theoretical Modeling, Washington, DC, United States, ${ }^{2}$ Neuronal Cytoskeletal Protein Regulation Section, National Institute of Neurological Disorders and Stroke, National Institutes of Health, Bethesda, $M D$, United States
\end{abstract}

Cellular localization, assembly and abnormal aggregation of neurofilaments depend on phosphorylation. Pathological processes associated with neurodegeneration exhibit aberrant accumulation of microtubule associated aggregated forms of hyperphosphorylated neuronal protein tau in cell bodies. These processes are critical for the disease progression in patients suffering from Alzheimer's disease, Parkinson's

OPEN ACCESS

Edited by:

Teresa Duda

Salus University, United States

Reviewed by:

Venkat Venkataraman,

Rowan University School of Osteopathic Medicine,

United States

Gustavo Fernando Pigino,

Instituto de Investigación Médica Mercedes y Martín Ferreyra (INIMEC),

Argentina

*Correspondence: Natalya Kurochkina info@schtm.org

Harish C. Pant

panth@ninds.nih.gov

Received: 02 June 2018 Accepted: 20 September 2018 Published: 08 October 2018

Citation:

Kurochkina N, Bhaskar M, Yadav SP and Pant HC (2018) Phosphorylation, Dephosphorylation, and Multiprotein Assemblies Regulate Dynamic Behavior of Neuronal Cytoskeleton: A Mini-Review.

Front. Mol. Neurosci. 11:373. doi: 10.3389/fnmol.2018.00373 disease, and Amyotrophic Lateral Sclerosis. In healthy cells, tau is localized in axons. Topographic regulation suggests that whereas the sites of synthesis of kinases and neurofilaments are the cell bodies, and sites of their functional assemblies are axons, phosphorylation/dephosphorylation are the key processes that arrange the molecules at their precise locations. Phosphorylation sites in the dynamic developmental and degenerative processes differ. Not all these processes are well understood. New advancements identify epigenetic factors involved in AD which account for the influence of age-related environment/genome interactions leading to the disease. Progress in proteomics highlights previously found major proteins and adds more to the list of those involved in AD. New key elements of specificity provide determinants of molecular recognition important for the assembly of macromolecular complexes. In this review, we discuss aberrant spatial distribution of neuronal polypeptides observed in neuropathies: aggregation, association with proteins of the neuronal cytoskeleton, and phosphorylation dependent dynamics. Particularly, we emphasize recent advancements in understanding the function and determinants of specific association of molecules involved in Alzheimer's disease with respect to the topographic regulation of phosphorylation in neuronal cytoskeleton and implications for the design of new therapies. Further, we address the role of various filament systems in maintenance of the shape, rigidity and dynamics of the cytoskeleton.

Keywords: phosphorylation, ankyrin repeats, protein structure, neurodegeneration, Alzheimer's disease

Abbreviations: $A \beta$, Amyloid- $\beta$; AD, Alzheimer's disease; AIS, axon initial segment; APP, amyloid precursor protein; CRMP, Collapsin response mediator protein; GSK-3 $\beta$, glycogen synthase kinase; DARPin, designed ankyrin repeats protein; MT, microtubule; NF, neurofilament; NMDAR, $N$-methyl-di-aspartate receptor; PD, Parkinson's disease; PIN1, peptidylprolyl cis/trans isomerase, NIMA-interacting 1; PP2A, protein phosphatase 2A; PSD95, postsynaptic density protein 95. 


\section{INTRODUCTION}

Cellular localization, assembly and abnormal aggregation of NFs depend on phosphorylation (Boumil et al., 2018). Pathological processes associated with neurodegeneration exhibit aberrant accumulation of MT associated aggregated forms of hyperphosphorylated neuronal protein tau in the cell bodies (Binukumar et al., 2013). These processes are critical for the disease progression in patients suffering from the AD, PD, and Amyotrophic Lateral Sclerosis (ALS). In the healthy cells, NFs and tau are localized in the axons. Proteolysis of the cell surface receptor and cell adhesion molecule APP results in the production of amyloid peptides (Roy and Rauk, 2005). A $\beta$ peptide induces alterations in axon to soma (retrograde) and soma to axon (anterograde) traffic of tau isoforms that regulates distribution of tau in neurons, F-actin remodeling, reduced MT dynamics, and impairment of astrocytic glutamate transport (Kanaan et al., 2013; Zempel et al., 2017). Distribution of NFs and tau is regulated by posttranslational modifications (Shukla et al., 2012; Kim et al., 2014) and interactions with cellular components such as AIS proteins ankyrin G (AnkG), EB1 and GSK-3 $\beta$ (Zempel et al., 2017) or PP2A and peptidyl-prolyl cis/trans isomerase PIN1 (Figure 1; Rudrabhatla et al., 2009). Tau and APP intracellular fragments alter gene transcription (Multhaup et al., 2015). Phosphorylation and dephosphorylation by kinases/phosphatases are key regulatory processes that we target to provide effective preventive and treatment options. AD brains are subject to neuroinflammation regulated by microglia and immune cells in the central nervous system (Mastroeni et al., 2017). Microglia carry out both beneficial and harmful functions: plaque removal or release of neurotoxic inflammatory materials. Although genetic associations exist which point to variations in genes of glia residing proteins, further studies identify epigenetic factors involved in $\mathrm{AD}$ which account for influence of agerelated environment/genome interactions leading to the disease. Progress in proteomics adds new proteins involved in $\mathrm{AD}$ (Gozal et al., 2009; Guzmán-Martinez et al., 2013; Robinson et al., 2017). Cytoskeletal proteins interact with each other and assemble in filaments in a highly specific manner; new key elements of specificity provide determinants of molecular recognition in the assembly of macromolecular complexes (Kurochkina and Iadarola, 2015). Understanding the function of kinases, phosphatases, and their targets outlines important implications and insights how to prevent and cure progressive neurological diseases.

In this review, we discuss aberrant spatial distribution of neuronal polypeptides observed in neuropathies: aggregation, association with proteins of the neuronal cytoskeleton, and phosphorylation dependent dynamics. Particularly, we emphasize recent advancements in understanding the function and determinants of specific association of the molecules involved in $\mathrm{AD}$ with respect to the topographic regulation of phosphorylation in neuronal cytoskeleton and implications for the design of new therapies. Further, we address the role of various filament systems in maintenance of the shape, rigidity and dynamics of the cytoskeleton.

\section{PHOSPHORYLATION AND DEPHOSPHORYLATION AS KEY FACTORS OF PROTEIN DYNAMICS, ASSOCIATION, NEURONAL DEVELOPMENT, AND PATHOLOGICAL PROCESSES}

\section{Cytoskeletal Proteins}

Filament systems like MTs, microfilaments, and intermediate filaments (IFs) comprise cell cytoskeleton as key elements regulating cell shape, rigidity and dynamics (Gruenbaum and Aebi, 2014). Structure of fibrous and globular filamentous proteins, assembly of filaments, and movement of motors are important for cell dynamics and function (Siddiqui and Straube, 2017). Cytoskeleton not only provides cell support but is also an important regulator of cell highly regular organization, mobility, reaction pathways, and response to extreme conditions (Unsain et al., 2018). Interactions of cytoskeletal components with each other and with surrounding proteins are highly specific, tightly regulated and drive functional assemblies of the multiprotein complexes (Kurochkina and Iadarola, 2015).

Studies of neuron specific cytoskeletal proteins such as tau and light (NFL), medium (NFM), and heavy (NFH) subunits of NFs show that phosphorylation plays important role in their function and dynamics. Phosphorylation stabilizes NFs, protects from proteolysis, and promotes calcium mediated assembly of NF subunits and cytoskeletal components in axons, whereas dephosphorylation results in more dynamic chains which repair and regenerate less mobile chains (Rudrabhatla et al., 2009; Boumil et al., 2018). Tau regulates MT polymerization and contains multiple binding sites. Its dynamic behavior is reflected in its structure. Large portions of the molecule are disordered (Figure 1) and are candidates for binding other ligands. Carrying PxxP sequence, tau mono-, di- and triphosphorylated peptides exhibit polyproline II conformation in complex with antibody (Figure 1A) and PIN1 (Figure 1B). Interactions of two Pin1 domains, WW substrate recognition and catalytic (Figure 1D), with each other, PP2A and other proteins contribute to its function (Wang et al., 2017). Tau obtains helical conformation upon binding to tubulin and F-actin (Kellogg et al., 2018; Figure 1C). All six human tau isoforms show common mechanism of tau/PAD (Figure 1F) conformational mobility associated with axonal transport inhibition (Cox et al., 2016). Both NFs and tau associate with MTs and produce huge complexes (Figure 1E) which are tethered to a membrane. Ankyrin repeats target proteins to the plasma membrane and link membrane proteins to the actin/spectrin cytoskeleton (Figure 2).

\section{Symmetry/Polarization in Neuronal Development}

Neuron-specific cytoskeleton gives neuronal cell its shape. Unique features of the neuron with cell body in the central and meters long axons in the peripheral nervous system require 
A

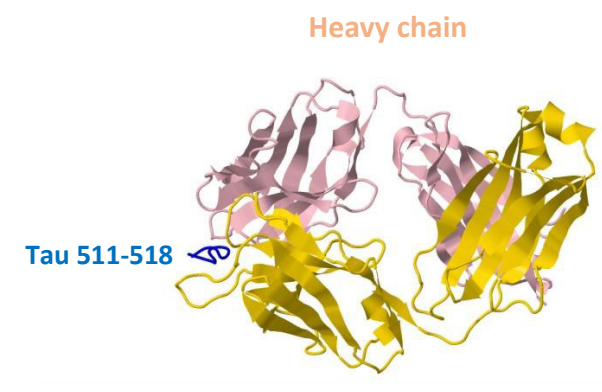

Light chain
C

B

D
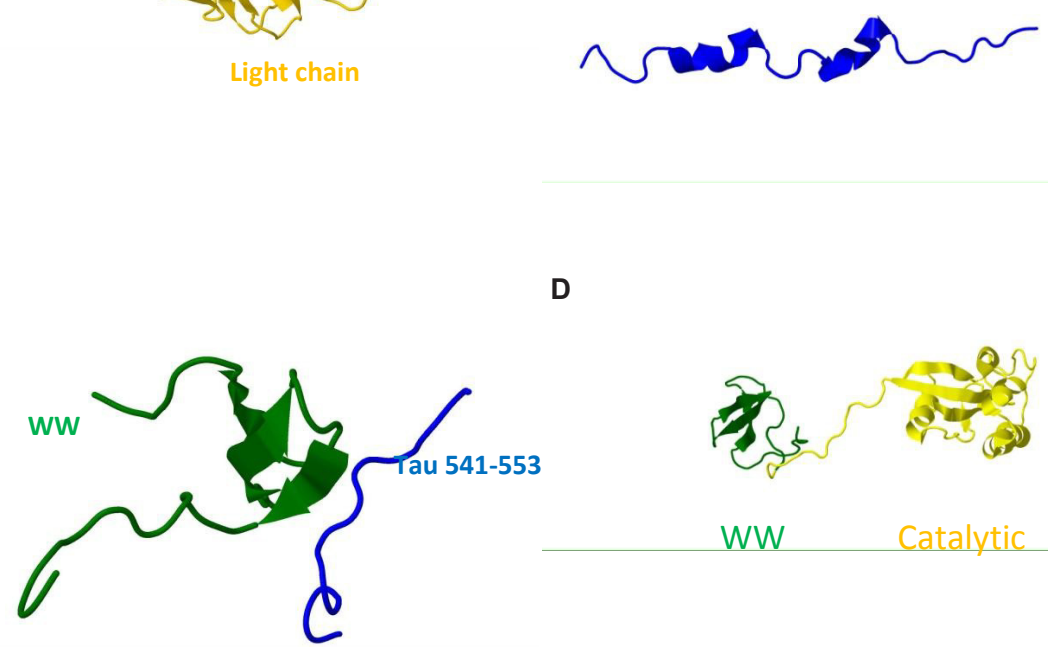

E

Tau 573-600

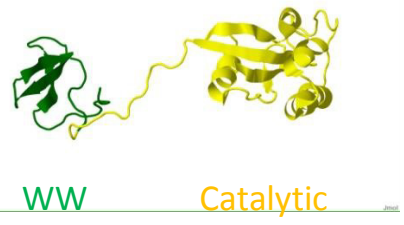

Tubulin

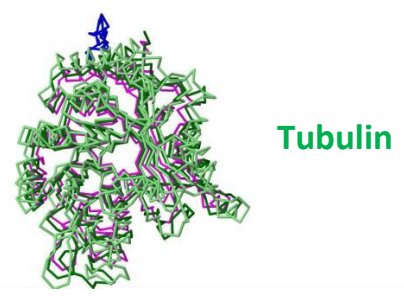

F

\begin{tabular}{|c|c|c|c|c|}
\hline PAD & $\mathrm{N}$ terminal & Proline rich & MT binding tandem repeats & C terminal \\
\hline 118 & 469 & $470 \quad 570$ & $571 \quad 685$ & $686 \quad 758$ \\
\hline
\end{tabular}

FIGURE 1 | Tau protein (A) Triply phosphorylated at S202/T205/S208 Tau peptide 202-209/511-518 (blue) in complex with anti-tau antibody AT8 (Light chain gold and Heavy chain pink) /Pdb entry 5e2W/. (B) Fragment 541-553 bound to the WW domain (green) of PIN1 / Pdb entry 1i8h/. (C) Fragments 292-319/608-635 /5n5b/ and 254-290/571-607/Pdb entry 5n5a/ bound to F-actin. (D) PIN1 WW (green) and catalytic (yellow) domains / Pdb entry 1nmv/. (E) Fragment 573-600 (blue) /Pdb entry 6cvn/ bound to tubulin (green, magenta). (F) Domain structure; PAD, Phosphatase Activating Domain.

specific structural components and maintenance (Binukumar et al., 2013). The asymmetric MT cytoskeleton plays a key role in axon-dendrite specification during the development and polarized protein sorting in mature neurons. The distribution of neuronal proteins to axons and dendrites depends on joint action of MT-binding proteins CRMP, directed motors (kinesin UNC104/Kif1A) (Kanaan et al., 2013), and diffusion barriers (ankyrin) (Maniar et al., 2012). 


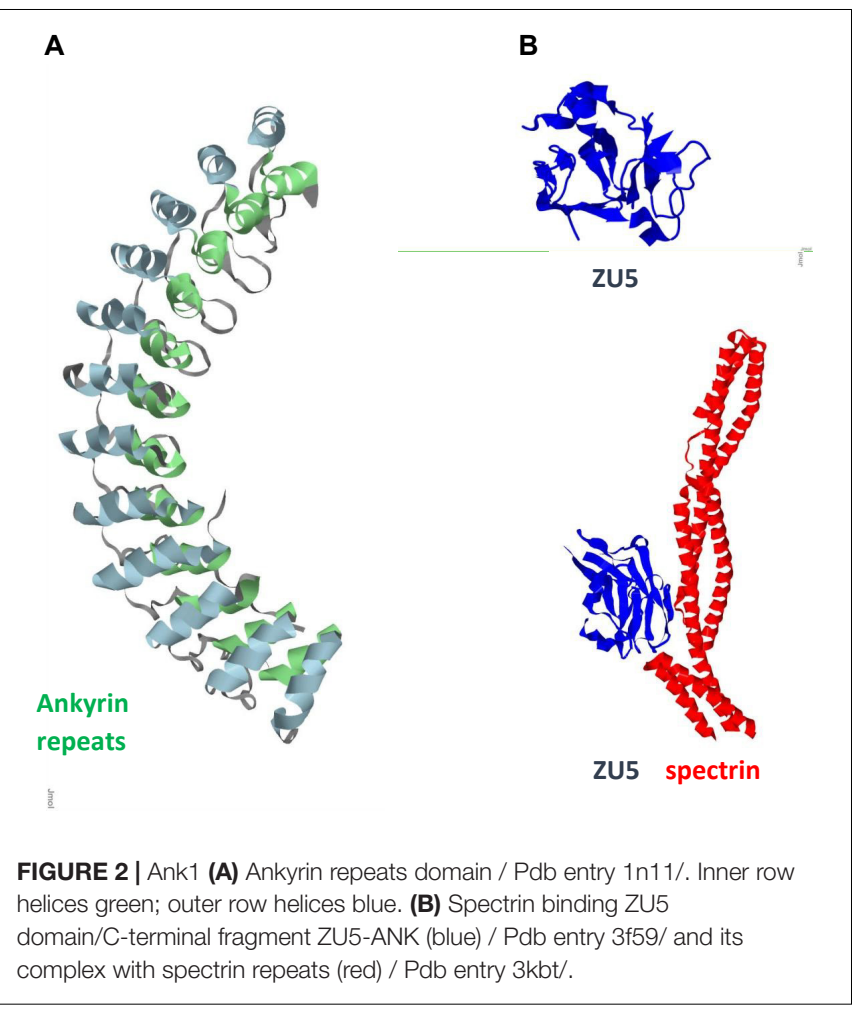

\section{Phosphorylation/Dephosphorylation in Pathologies}

Pathological hyperphosphorylated and aggregated tau is surrounded by more than 150 proteins as shown by studies of neurofibrillary tangles (Wang et al., 2005). Many efforts focus on abnormal aggregation of $A \beta, N F s$ and tau. However, the impairment of the function of tau, NFs, and other proteins in topographic regulation of phosphorylation could provide keys to pathology (Lane et al., 2012). Tau and NF colocalize and co-immunoprecipitate with PSD95 regulating the interaction of PSD95 with NMDAR subunits. Axonal protein tau has a dendritic function: postsynaptic targeting of the Src kinase Fyn. Fyn phosphorylates the NR subunit 2 of NMDA receptors (NMDARs) and promotes its interaction with the PSD95 (Ittner et al., 2010). NMDARs, for instance, mediate excitatory glutamatergic signals from ON and OFF bipolar cells in distinct subliminae of the inner plexiform layer to retinal ganglion cells. Tetrameric structure of NMDAR embraces two NR1 and two NR2 subunits. Localization of NR2A/NR1C2 splice variant and PSD93 /PSD95 of MAGUK to the PSD OFF synapses whereas NR2B/NR1C2 splice variant and SAP102/MAGUK to the ON synapses perisynaptically suggests specific roles for various receptor isoforms (Zhang et al., 2016). NMDAR is regulated by Cdk5 phosphorylation ( $\mathrm{Li}$ et al., 2001). MT-binding protein CRMP (UNC-33) being similar to tau is also involved in dynamic regulation of MTs, and its isoforms expressed during brain oncogenesis carry out signaling function in axon outgrowth and guidance (Bretin et al., 2005; Meng et al., 2016). Cdk5, GSK3 $\beta$, and Pin1 regulate CRMP2A, major isoform of CRMP (Maniar et al., 2012). Unlike tau, CRMP2, regulated by posttranslational modifications, actively engages in protein endocytosis and vesicular cycling. A $\beta$-induced modifications of CRMP2 could possibly result in impaired axonal transport and synapse loss: pathological processes associated with $\mathrm{AD}$ (Hensley and Kursula, 2016; Yang et al., 2017). CRMP2 is targeted as possible agent that could prevent or delay the progression of AD.

Hyperphosphorylation with involvement of Cdk5, GSK3 $\beta$ and other kinases and oxidation contribute to $\mathrm{AD}$ pathogenesis (Khanna et al., 2012). Phosphorylation of neuronal cytoskeletal proteins is restricted selectively in axonal but not in cell body compartment under physiological conditions. However, under pathological state, it is deregulated, and occurs aberrantly in the cell body compartment as well. Phosphorylation sites in the dynamic developmental and degenerative processes differ ( $\mathrm{Yu}$ et al., 2009). Strategies to treat pathologies target posttranslational modifications and mediation of localization, dynamics, and assembly.

\section{DOMAINS OF SPECIFIC MULTIPROTEIN ASSEMBLIES: ANKYRIN REPEATS}

Ankyrin repeats are important scaffold components that mediate shape, rigidity, dynamics of association, and function of the multiprotein assemblies. In mammals, ankyrins are encoded by three different genes, ankyrin $\mathrm{R}$ (ANK1), ankyrin $\mathrm{B}$ (ANK2/ANKB), and ankyrin G (ANK3/ANKG), all of which have similar structure and function, and undergo alternative splicing to generate multiple isoforms (Kordeli et al., 1995). Epigenetic wide association studies identified ANK1 (Figure 2) as one of the key risk factors for $\mathrm{AD}$ (Chi et al., 2016; Mastroeni et al., 2017). ANK1 is also implicated in type 2 diabetes (Imamura et al., 2012). Microglia is the source of ANK1 and no elevated expression of ANK1 occurs in CA1 astrocytes or neurons. Changes in expression of 13 out of 14 ankyrin repeats proteins in $\mathrm{AD}$ neurons with downregulated AnkRD18 and upregulated AnkRD34, increased ANK1 in PD microglia, elevated AnkRD36/ANKRD52/ANKRD18CP in AD astrocytes, and overexpressed Ankle2 in PD microglia suggest that this involvement is not disease specific but a process related to neuroinflammation. Association of ANK1 gene methylation changes with AD (Lunnon et al., 2014) shows how epigenetic mechanisms could provide a link to age-related inflammation and highlights this gene as an $\mathrm{AD}$ risk factor.

Progress in proteomics reveals AnkB and AnkG involvement in AD (Gozal et al., 2009). Giant long ANKB isoform in Drosophila shows involvement in synapse stability and links to neurodegeneration (Pielage et al., 2008). Knockout of the long AnkG isoform results in neuroanatomical defects in mice (van der Werf et al., 2017) and impaired filtering of cellular components (Sun et al., 2017). AnkG is the site of clustering of $\mathrm{Na}^{+}$and $\mathrm{K}^{+}$channels and their interactions with numerous modulators (Gennarini et al., 2017). AnkG, site of assembly of $\mathrm{Na}^{+} / \mathrm{K}^{+}$-ATPase, together with other cytoskeletal proteins (GABA A receptor gephyrin) makes connections to membrane proteins associated with lipid rafts (Dalskov et al., 2005). 
AnkG may be considered the most important AIS constituent. The AIS is a specialized compartment in neurons that resides between axonal and somatodendritic domains where the assembly of neuronal IFs takes place. It serves as a site of action potential firing and helps maintain neuron polarity. It also acts as a submembrane diffusion barrier that restrict the mobility of plasma membrane components (Bennett, 1992; Winckler et al., 1999) and an intracellular selective filter for the transport of organelles and molecules between these domains through the cytoplasm. AnkG integrates proteins that function in three layers of the AIS: the plasma membrane (outermost surface), submembrane cytoskeleton (middle layer), and inner AIS shaft (cytoplasmic region), and acts as its main organizer. Advancements in deciphering structure of ankyrins, phosphorylation states and role in assembly of the multiprotein complexes help to understand the mechanisms and suggest new studies (Zhang et al., 1998; Chen et al., 2017).

\section{CONCLUSION}

Progress in understanding the role of phosphorylation/ dephosphorylation in regulation of spatial distribution and transport of proteins involved in neurodegenerative diseases brings new approaches and evolvement of new treatments.

\section{REFERENCES}

Bennett, V. (1992). Ankyrins. Adaptors between diverse plasma membrane proteins and the cytoplasm. J. Biol. Chem. 267, 8703-8706.

Binukumar, B. K., Shukla, V., Amin, N. D., Reddy, P., Skuntz, S., Grant, P., et al. (2013). Topographic regulation of neuronal intermediate filaments by phosphorylation, role of peptidyl-prolyl isomerase 1: significance in neurodegeneration. Histochem. Cell Biol. 140, 23-32. doi: 10.1007/s00418-0131108-7

Boumil, E. F., Vohnoutka, R., Lee, S., Pant, H., and Shea, T. B. (2018). Assembly and turnover of neurofilaments in growing axonal neurites. Biol. Open 7:bio028795. doi: 10.1242/bio.028795

Bretin, S., Reibel, S., Charrier, E., Maus-Moatti, M., Auvergnon, N., Thevenoux, A., et al. (2005). Differential expression of CRMP1, CRMP2A, CRMP2B, and CRMP5 in axons or dendrites of distinct neurons in the mouse brain. J. Comp. Neurol. 486, 1-17. doi: 10.1002/cne.20465

Chen, K., Li, J., Wang, C., Wei, Z., and Zhang, M. (2017). Autoinhibition of ankyrin-B/G membrane target bindings by intrinsically disordered segments from the tail regions. eLife 6:e29150. doi: 10.7554/eLife.29150

Chi, S., Song, J. H., Tan, M. S., Zhang, W., Wang, Z. X., Jiang, T., et al. (2016). Association of single-nucleotide polymorphism in ANK1 with lateonset Alzheimer's Disease in Han Chinese. Mol. Neurobiol. 53, 6476-6481. doi: 10.1007/s12035-015-9547-x

Cox, K., Combs, B., Abdelmesih, B., Morfini, G., Brady, S. T., and Kanaan, N. M. (2016). Analysis of isoform-specific tau aggregates suggests a common toxic mechanism involving similar pathological conformations and axonal transport inhibition. Neurobiol. Aging 47, 113-126. doi: 10.1016/j.neurobiolaging.2016. 07.015

Dalskov, S. M., Immerdal, L., Niels-Christiansen, L. L., Hansen, G. H., Schousboe, A., and Danielsen, E. M. (2005). Lipid raft localization of GABA A receptor and $\mathrm{Na}+, \mathrm{K}+-$-ATPase in discrete microdomain clusters in rat cerebellar granule cells. Neurochem. Int. 46, 489-499. doi: 10.1016/j.neuint. 2004.11.010

Gennarini, G., Bizzoca, A., Picocci, S., Puzzo, D., Corsi, P., Furley, A., et al. (2017). The role of Gpi-anchored axonal glycoproteins in neural development and
Understanding MT dynamics, posttranslational modifications, specific distribution and assembly of cellular components, and signaling which links neuronal dysfunction to neuropathies leads to the development of new therapies (Yeh et al., 2004; Shukla et al., 2012; Kanaan et al., 2013). A $\beta$ accumulation can be treated with amyloid removal strategies such as one utilizing DARPins (Hanenberg et al., 2014). Ankyrin G vaccination is tested for the reduction of $\mathrm{A} \beta$ and lowering toxicity (Santuccione et al., 2013). Ankyrin repeats are interaction sites of phosphorylation dependent dynamic assembly of proteins and nucleic acids (Hall et al., 2018) including those involved in transcription regulation (Kohda et al., 2016) and signaling, and present promising targets for the design of new drugs.

\section{AUTHOR CONTRIBUTIONS}

All authors listed have made a substantial, direct and intellectual contribution to the work, and approved it for publication.

\section{FUNDING}

This work was supported by NIH intramural funding and Funding Program of The School of Theoretical Modeling.

neurological disorders. Mol. Cell. Neurosci. 81, 49-63. doi: 10.1016/j.mcn.2016. 11.006

Gozal, Y. M., Duong, D. M., Gearing, M., Cheng, D., Hanfelt, J. J., Funderburk, C., et al. (2009). Proteomics analysis reveals novel components in the detergentinsoluble subproteome in Alzheimer's disease. J. Proteome Res. 8, 5069-5079. doi: $10.1021 /$ pr900474t

Gruenbaum, Y., and Aebi, U. (2014). Intermediate filaments: a dynamic network that controls cell mechanics. F1000Prime Rep. 6:54. doi: 10.12703/P6-54

Guzmán-Martinez, L., Farías, G. A., and Maccioni, R. B. (2013). Tau oligomers as potential targets for Alzheimer's diagnosis and novel drugs. Front. Neurol. 4:167. doi: 10.3389/fneur.2013.00167

Hall, B. E., Prochazkova, M., Sapio, M. R., Minetos, P., Kurochkina, N., Binukumar, B. K., et al. (2018). Phosphorylation of the transient receptor potential Ankyrin 1 by cyclin-dependent kinase 5 affects chemo-nociception. Sci. Rep. 8:1177. doi: 10.1038/s41598-018-19532-6

Hanenberg, M., McAfoose, J., Kulic, L., Welt, T., Wirth, F., Parizek, P., et al. (2014). Amyloid- $\beta$ peptide-specific DARPins as a novel class of potential therapeutics for Alzheimer disease. J. Biol. Chem. 289, 27080-27089. doi: 10.1074/jbc.M114. 564013

Hensley, K., and Kursula, P. (2016). Collapsin response mediator protein-2 (CRMP2) is a plausible etiological factor and potential therapeutic target in Alzheimer's disease: comparison and contrast with microtubule-associated protein tau. J. Alzheimers Dis. 53, 1-14. doi: 10.3233/JAD-160076

Imamura, M., Maeda, S., Yamauchi, T., Hara, K., Yasuda, K., Morizono, T., et al. (2012). Diabetes genetics replication and meta-analysis (DIAGRAM) consortium, Nakamura Y, Kadowaki T. A single-nucleotide polymorphism in ANK1 is associated with susceptibility to type 2 diabetes in Japanese populations. Hum. Mol. Genet. 21, 3042-3049. doi: 10.1093/hmg/dds113

Ittner, L. M., Ke, Y. D., Delerue, F., Bi, M., Gladbach, A., van Eersel, J., et al. (2010). Dendritic function of tau mediates amyloid-beta toxicity in Alzheimer's disease mouse models. Cell 142, 387-397. doi: 10.1016/j.cell.2010.06.036

Kanaan, N. M., Pigino, G. F., Brady, S. T., Lazarov, O., Binder, L. I., and Morfini, G. A. (2013). Axonal degeneration in Alzheimer's disease: when signaling abnormalities meet the axonal transport system. Exp. Neurol. 246, 44-53. doi: 10.1016/j.expneurol.2012.06.003 
Kellogg, E. H., Hejab, N. M. A., Poepsel, S., Downing, K. H., DiMaio, F., and Nogales, E. (2018). Near-atomic model of microtubule-tau interactions. Science 360, 1242-1246. doi: 10.1126/science.aat1780

Khanna, R., Wilson, S. M., Brittain, J. M., Weimer, J., Sultana, R., Butterfield, A., et al. (2012). Opening Pandora's jar: a primer on the putative roles of CRMP2 in a panoply of neurodegenerative, sensory and motor neuron, and central disorders. Future Neurol. 7, 749-771. doi: 10.2217/fnl.12.68

Kim, B. M., You, M. H., Chen, C. H., Lee, S., Hong, Y., Hong, Y., et al. (2014). Death-associated protein kinase 1 has a critical role in aberrant tau protein regulation and function. Cell Death Dis. 5:e1237. doi: 10.1038/cddis.2014.216

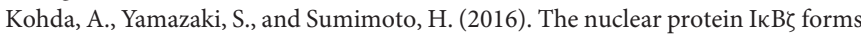
a transcriptionally active complex with nuclear factor- $\kappa \mathrm{B}(\mathrm{NF}-\kappa \mathrm{B}) \mathrm{p} 50$ and the Lcn 2 promoter via the N- and C-terminal Ankyrin repeat Motifs. J. Biol. Chem. 291, 20739-20752. doi: 10.1074/jbc.M116.719302

Kordeli, E., Lambert, S., and Bennett, V. (1995). AnkyrinG. A new ankyrin gene with neural-specific isoforms localized at the axonal initial segment and node of Ranvier. J. Biol. Chem. 270, 2352-2359. doi: 10.1074/jbc.270.5.2352

Kurochkina, N. A., and Iadarola, M. J. (2015). Helical assemblies: structure determinants. J. Theor. Biol. 369, 80-84. doi: 10.1016/j.jtbi.2015.01.012

Lane, R. F., Shineman, D. W., Steele, J. W., Lee, L. B., and Fillit, H. M. (2012). Beyond amyloid: the future of therapeutics for Alzheimer's disease. $A d v$. Pharmacol. 64, 213-271. doi: 10.1016/B978-0-12-394816-8.00007-6

Li, B. S., Sun, M. K., Zhang, L., Takahashi, S., Ma, W., Vinade, L., et al. (2001). Regulation of NMDA receptors by cyclin-dependent kinase-5. Proc. Natl. Acad. Sci. U.S.A. 98, 12742-12747. doi: 10.1073/pnas.211428098

Lunnon, K., Smith, R., Hannon, E., De Jager, P. L., Srivastava, G., Volta, M., et al. (2014). Methylomic profiling implicates cortical deregulation of ANK1 in Alzheimer's disease. Nat. Neurosci. 17, 1164-1170. doi: 10.1038/nn.3782

Maniar, T. A., Kaplan, M., Wang, G. J., Shen, K., Wei, L., Shaw, J. E., et al. (2012). Unc-33/CRMP and ankyrin organize microtubules and localize kinesin to polarize axon-dendrite sorting. Nat. Neurosci. 15, 48-56. doi: 10.1038/nn.2970

Mastroeni, D., Sekar, S., Nolz, J., Delvaux, E., Lunnon, K., Mill, J., et al. (2017). ANK1 is up-regulated in laser captured microglia in Alzheimer's brain; the importance of addressing cellular heterogeneity. PLoS One 12:e0177814. doi: 10.1371 /journal.pone.0177814

Meng, L., Chen, C., and Yan, D. (2016). Regulation of gap junction dynamics by UNC-44/ankyrin and UNC-33/CRMP through VAB-8 in C. elegans neurons. PLoS Genet. 12:e1005948. doi: 10.1371/journal.pgen.1005948

Multhaup, G., Huber, O., Buée, L., and Galas, M. C. (2015). Amyloid Precursor Protein (APP) metabolites APP intracellular fragment (AICD), Aß42, and Tau in nuclear roles. J. Biol. Chem. 290, 23515-23522. doi: 10.1074/jbc.R115.677211

Pielage, J., Cheng, L., Fetter, R. D., Carlton, P. M., Sedat, J. W., and Davis, G. W. (2008). A presynaptic giant ankyrin stabilizes the NMJ through regulation of presynaptic microtubules and transsynaptic cell adhesion. Neuron 58, 195-209. doi: 10.1016/j.neuron.2008.02.017

Robinson, R. A., Amin, B., and Guest, P. C. (2017). Multiplexing biomarker methods, proteomics and considerations for Alzheimer's disease. Adv. Exp. Med. Biol. 974, 21-48. doi: 10.1007/978-3-319-52479-5_2

Roy, S., and Rauk, A. (2005). Alzheimer's disease and the 'ABSENT' hypothesis: mechanism for amyloid beta endothelial and neuronal toxicity. Med. Hypotheses 65, 123-137. doi: 10.1016/j.mehy.2004.08.031

Rudrabhatla, P., Albers, W., and Pant, H. C. (2009). Peptidyl-Prolyl Isomerase 1 regulates protein phosphatase $2 \mathrm{~A}$-mediated topographic phosphorylation of neurofilament proteins. J. Neurosci. 29, 14869-14880. doi: 10.1523/ JNEUROSCI.4469-09.2009

Santuccione, A. C., Merlini, M., Shetty, A., Tackenberg, C., Bali, J., Ferretti, M. T., et al. (2013). Active vaccination with ankyrin $G$ reduces $\beta$-amyloid pathology in APP transgenic mice. Mol. Psychiatry 18, 358-368. doi: 10.1038/mp.2012.70
Shukla, V., Skuntz, S., and Pant, H. C. (2012). Deregulated Cdk5 activity is involved in inducing Alzheimer's disease. Arch. Med. Res. 43, 655-662. doi: 10.1016/j. arcmed.2012.10.015

Siddiqui, N., and Straube, A. (2017). Intracellular cargo transport by kinesin-3 motors. Biochemistry 82, 803-815. doi: 10.1134/S0006297917070057

Sun, X., Wu, Y., Gu, M., Liu, Z., Ma, Y., Li, J., et al. (2017). Selective filtering defect at the axon initial segment in Alzheimer's disease mouse models. Proc. Natl. Acad. Sci. U.S.A. 111, 14271-14276. doi: 10.1073/pnas.1411837111

Unsain, N., Stefani, F. D., and Cáceres, A. (2018). The actin/spectrin membraneassociated periodic skeleton in neurons. Front. Synaptic Neurosci. 10:10. doi: 10.3389/fnsyn.2018.00010

van der Werf IM, Van, Dam D, Missault, S., Yalcin, B., De, Deyn PP, Vandeweyer, G., et al. (2017). Behavioural characterization of AnkyrinG deficient mice, a model for ANK3 related disorders. Behav. Brain Res. 328, 218-226. doi: 10.1016/j.bbr. 2017.04.014

Wang, J., Kawasaki, R., Uewaki, J. I., Rashid, A. U. R., Tochio, N., and Tate, S. I. (2017). Dynamic allostery modulates catalytic activity by modifying the hydrogen bonding network in the catalytic site of human Pin1. Molecules 22:E992. doi: 10.3390/molecules22060992

Wang, Q., Woltjer, R. L., Cimino, P. J., Pan, C., Montine, K. S., Zhang, J., et al. (2005). Proteomic analysis of neurofibrillary tangles in Alzheimer disease identifies GAPDH as a detergent-insoluble paired helical filament tau binding protein. FASEB J. 19, 869-871. doi: 10.1096/fj.04-3210fje

Winckler, B., Forscher, P., and Mellman, I. (1999). A diffusion barrier maintains distribution of membrane proteins in polarized neurons. Nature 397, 698-701. doi: $10.1038 / 17806$

Yang, Z., Kuboyama, T., and Tohda, C. A. (2017). Systematic strategy for discovering a therapeutic drug for Alzheimer's disease and its target molecule. Front. Pharmacol. 19:340. doi: 10.3389/fphar.2017.00340

Yeh, T. S., Hsieh, R. H., Shen, S. C., Wang, S. H., Tseng, M. J., Shih, C. M., et al. (2004). Nuclear betaII-tubulin associates with the activated notch receptor to modulate notch signaling. Cancer Res. 64, 8334-8340. doi: 10.1158/0008-5472. CAN-04-2197

Yu, Y., Run, X., Liang, Z., Li, Y., Liu, F., Liu, Y., et al. (2009). Developmental regulation of tau phosphorylation, tau kinases, and tau phosphatases. J. Neurochem. 108, 1480-1494. doi: 10.1111/j.1471-4159.2009.05882.x

Zempel, H., Dennissen, F. J. A., Kumar, Y., Luedtke, J., Biernat, J., Mandelkow, E. M., et al. (2017). Axodendritic sorting and pathological missorting of Tau are isoform-specific and determined by axon initial segment architecture. J. Biol. Chem. 292, 12192-12207. doi: 10.1074/jbc.M117.784702

Zhang, J., Petralia, R. S., and Wang, Y. X. (2016). Diamond JS. High-resolution quantitative immunogold analysis of membrane receptors at retinal ribbon synapses. J. Vis. Exp. 108:53547. doi: 10.3791/53547

Zhang, Z., Devarajan, P., Dorfman, A. L., and Morrow, J. S. (1998). Structure of the ankyrin-binding domain of alpha-Na,K-ATPase. J. Biol. Chem. 273, 18681-18684. doi: $10.1074 /$ jbc.273.30.18681

Conflict of Interest Statement: The authors declare that the research was conducted in the absence of any commercial or financial relationships that could be construed as a potential conflict of interest.

Copyright (C) 2018 Kurochkina, Bhaskar, Yadav and Pant. This is an open-access article distributed under the terms of the Creative Commons Attribution License (CC BY). The use, distribution or reproduction in other forums is permitted, provided the original author(s) and the copyright owner(s) are credited and that the original publication in this journal is cited, in accordance with accepted academic practice. No use, distribution or reproduction is permitted which does not comply with these terms. 